\title{
Songmoo Kho 1947-1993
}

Der koreanische Sprachwissenschaftler und Kulturhistoriker Dr. Songmoo Kho (Ko Songmu) wurde am 3.12.1947 in der ländlichen Kleinstadt Keum-San, Chung-Nam, südlich von Seoul, geboren. Sein Vater war der bekannteste Arzt der Ortschaft und im Anschluß an das Haus der Familie betrieb er eine eigene kleine Klinik zusammen mit einer Apotheke. Das Haus des Großvaters befand sich ebenfalls in derselben Häusergruppe. Ungefähr $30 \mathrm{~km}$ entfernt im Gebirge lag eine vom Großvater angelegte Waldparzelle.

Während des Koreakrieges hatte der türkische Präsident Celal Bayar freiwillige Truppen für die Vereinten Nationen nach Korea entsandt. Der kleine Songmoo lernte den alten Imam eines türkischen Truppenverbandes kennen und befreundete sich mit ihm. Der Alte schenkte Songmoo einen arabischen Koran, wodurch sich dieser stets zum Islam hingezogen fühlte.

Schon in Korea begann Songmoo sich für Sprachen und Völker zu interessieren. Über die Frage einer möglichen Zusammenhörigkeit des Koreanischen mit den sogenannten altaischen Sprachen grübelte er häufig nach, besonders als sich nach der 30jährigen japanischen Okkupation und dem überstandenen Krieg endlich die nationalen Wissenschaften frei etablieren und entwickeln konnten. Dies führte ihn unmittelbar zu den Werken von Professor G. J. Ramstedt (1873-1950), einem der Begründer der modernen Mongolistik und vergleichenden Altaistik. Während seines zehnjährigen Dienstes als Finnlands chargé d'affaires in Japan in den Jahren 1919-29 hatte Ramstedt zuerst Japanisch gelernt und dann mit dem jungen koreanischen Studenten Ryu Cinkel Koreanisch studiert. Das Koreanische wurde Ramstedts große Liebe für den Rest seines Lebens und resultierte in einer bahnbrechenden modernen Grammatik (A Korean Grammar. MSFOu LXXXII, Helsinki 1939; neu gedruckt in Korea 1981) und einem altaisch ausgerichteten etymologischen Wörterbuch (Studies in Korean Etymology I-II. MSFOu XCV, 1949; XCV: 2, 1952).

Also beschloß Songmoo als junger Student i. J. 1967 nach Finnland zu reisen, um hier zu studieren. Erste Kontakte fand er zu einer Bauemfamilie in Tohmajărvi, in Finnisch-Karelien, und auch nach seiner Etablierung in Helsinki besuchte er diese Familie häufig wăhrend des Sommers. Am schwierigsten war es für Songmoo, in einem Bett schlafen zu lernen - er fürchtete lange Zeit, hilflos hinauszufallen. 
An der Universität Helsinki studierte Songmoo bei Prof. Dr. Aulis J. Joki Finnougristik, bei Prof. Dr. Pentti Aalto Altaistik und bei Prof. Dr. Jussi Aro Arabistik. Daneben besuchte er Kurse in vielen anderen Sprachen und lernte u. a. Finnisch, Estnisch, Ungarisch, Türkisch, Neugriechisch und Russisch sprechen. Er legte auch ein Examen in Assyriologie (Hauptfach Sumerisch) ab. Als mit dem Islam verknüpfte Sprachen schätzte er Türkisch, Arabisch und Persisch besonders. Nachdem er einst Professor Joki im Institut für finnischugrische Sprachen (Castrenianum) besucht hatte, meinte man dort, daß man nun gewiß einen Korjaken vor sich gehabt hätte!

Nach vier Jahren in Finnland besuchte Songmoo sein Elternhaus erst wieder im Herbst 1971. Nach dem Mondkalender feierte man seinen Geburtstag am 9. Dezember. Die Eltern bereiteten für ihn, »den Sohn, der vier Jahre lang von zu Hause weggewesen war», Ochsenkotlett, Eisbein und mantu. Songmoo konnte nicht wieder abreisen, denn der Kriegsgefahr wegen wurde der Notstand erklärt; er mußte befürchten, zwecks Militärdienst in Korea bleiben zu müssen. Während dieser Zeit hielt er zwei Vorträge über das Verhältnis der uralischen Sprachen zum Koreanischen - etwas unerwartet nach Ramstedts altaisch-koreanischer Arbeit, obgleich man in Korea die ural-altaische Hypothese allgemein akzeptierte. Songmoos Standpunkt war in Korea vollkommen unbekannt, da alle Gelehrten sich Ramstedts altaisch-koreanischen Ideen, die sogar in einem Lehrbuch für die Mittelschule erwähnt sind, anschlossen. "Die Alten wissen nichts und nicken nur mit dem Kopf", schreibt er. - Er selbst lehnte die in Korea populäre mandschu-koreanische Verwandtschaftshypothese vollkommen $\mathrm{ab}$ und befürwortete später sogar die anti-altaischen Gesichtspunkte von G. Doerfer, G. Clauson usw. Einmal ging Songmoo zu einem koreanischen Professor und fragte diesen etwas. Jener erkundigte sich nach seinem Alter und gab ihm den Rat, seine Vorlesungen zu besuchen.

Um das Finnische nicht zu vergessen, bat er die finnische Botschaft in Tokio, ihm Tageszeitungen zuzuschicken. Im Frühjahr 1972 konnte er endlich wieder nach Finnland zurückkehren und im Jahre 1973 bestand er das Abschlußexamen in seinem Hauptfach Finnougristik. Seine Examensarbeit (Korean kielen ja uralilaisten kielten välinen etymologinen vertailu. 59 S.; eximia cum laude approbatur) schrieb er über jene etwa 60 koreanischen Wörter, für die er genaue sprachhistorische Entsprechungen in den uralischen Sprachen gefunden hatte. Er vertrat die Ansicht, daß zwischen den Sprechern des Koreanischen und der uralischen Sprachen eine starke gegenseitige Wechselbeziehung bestanden haben müsse. 1974 studierte Songmoo Ungarisch an der Sommeruniversität von Debrecen.

In den Jahren 1978-80 mußte Songmoo wieder für zwei Jahre in Korea verweilen. Er hatte eine finnische Ministerdelegation begleitet und durfte zu seiner großen Enttäuschung nicht wieder ausreisen. Seinen eigenen Plänen zufolge benötigte er noch ein Jahr, um in Finnland zu studieren, aber wegen sei- 
ner seltenen Osteuropa-Kenntnisse bot man ihm eine Stellung als Experte in einem neuen arealwissenschaftlichen Institut in Seoul mit einem einjährigen Vertrag an. In dieser Situation schrieb er, daß er viel lieber als Akademiker tätig geworden wäre; ideal wäre für ihn ein Leben als freier Wissenschaftler auf eigene Kosten gewesen. Als freiwilliger Repräsentant Finnlands versuchte er energisch, die wechselseitigen Interessen zu fördem. Das verunglückte KAL-Flugzeug führte dazu, daß man seine Kenntnisse über Sowjetkarelien bald auch in der Öffentlichkeit benötigte. Vor sprachwissenschaftlichen Vereinigungen hielt er Vorträge über Uralistik; Kurse in Finnisch oder Ungarisch brauchte hingegen niemand.

Er ließ sich dann alle seine Sachen und Bücher aus Finnland nachschicken und sagte zynisch: »Das ist der Schlußpunkt meiner zehnjährigen Reise.» Er empfing Freundesbesuche aus Finnland, traf alte Schulkameraden und erachtete es als notwendig, auf eine Dissertation hinzuzielen. Er hatte nämlich an mehreren koreanischen Universitäten versucht, eine Stellung zu finden, sein Fach wurde aber als allzu exklusiv angesehen; ohne Doktorexamen und weiterführende Studien in Korea stand er außerhalb der akademischen Welt Koreas. Er fühlte sich fremd und begann über eine Diplomatenkarriere in Osteuropa nachzudenken. Die koreanische Diplomatie arbeitete während dieser Zeit daraufhin, diplomatische Kontakte zur Sowjetunion und zu den anderen osteuropäischen Staaten zu knüpfen; wie wäre also eine Stellung als erster koreanischer Botschafter in Budapest...?

Songmoo beklagte sich bei seinen Freunden, daß man in Korea sein Leben als verloren ansah, da er studiert hatte, sogar im Ausland, aber ohne Amt und Würden geblieben war. Man sagte ihm immer, daß man etwas sein müsse, daß man sein Talent zeigen müsse.

Schließlich bat Songmoo darum, ihm eine Einladung zu besorgen, um wieder für zwei Jahre nach Finnland kommen zu können und hier eine Dissertation über sibirisch-koreanische Kontakte (unter dem Aspekt Sibirien als Urheimat der Koreaner) schreiben zu können. Es wurde besonders betont, daß die Universität Helsinki ihn dringend benötigte als Lehrer des Koreanischen, wofür er bereits 1972-77 verantwortlich gewesen war. Dieser Plan konnte leider nicht verwirklicht werden, es blieben jedoch die Träume: Alte Leute sagten $\mathrm{ihm}$, daß man im Leben alles positiv sehen und langsam angehen solle.

Im November 1979 verließ Songmoo seine Stellung in Seoul. Bald darauf besuchte Professor Pentti Aalto Korea und hielt dort einen Vortrag. Nachdem der Entschluß gefaßt war, wieder nach Finnland zurückzukehren, überließ Songmoo einen großen Teil seiner Bücher dem Hangeul-Verein und erhielt dafür wichtige Serienpublikationen für die Orientalia-Bibliothek der Universität Helsinki. Im Frühjahr 1980 stand er wieder auf finnischem Boden. Er setzte nun für zehn Jahre seinen Sprachunterricht fort und erteilte dabei auch mehrere Kurse gratis. Unter seinen Kollegen unterrichtete auch Dr. Emine Gürsoy-Nas- 
kali, eine Enkelin des erwähnten türkischen Präsidenten Celal Bayar, in den Jahren 1977-84 Türkisch.

1986 legte Songmoo Kho das Lizentiaten-Examen ab mit der Arbeit Koreans in Soviet Central Asia und promovierte 1987 mit einer hieraus weiterentwickelten Dissertation des gleichen Titels (veröffentlicht in Studia Orientalia $61.262 \mathrm{~S}$.).

1987-91 war Songmoo Kho verantwortlich für den neugegründeten Lehrstuhl für ostasiatische Studien an der Universität Helsinki; er wurde damit zum eigentlichen Bahnbrecher für eine Ostasien-Tradition in Finnland. Als erster Vertreter dieser Professur konnte er junge Begabungen mittels seiner persönlichen Kontakte in den fernen Osten schicken, bildete er die ersten Finnen mit guten Kentnissen im Koreanischen aus und vermehrte markant die Anzahl jener, die ihre praktischen Fertigkeiten in China, Taiwan oder Japan erworben hatten. Im August-September 1988 arbeitete er bei den Olympischen Spielen in Seoul für den Finnischen Rundfunk als Redakteur, Dolmetscher und offizielle Kontaktperson.

Songmoo Kho gehörte zu den stützenden Kräften des Instituts für asiatische und afrikanische Studien an der Universität Helsinki, und zwar seit dessen Anfängen im Jahre 1973. Ohne seine eminente Hilfsbereitschaft hätten manche Dinge nie durchgeführt werden können. Er besaß eine heitere, offene Gemütsart, die von seinem weiten Freundeskreis hochgeschătzt wurde. Wo immer er hinkam, verwandelte er die Atmosphäre in etwas Angenehmes, Positives und Ungezwungenes. Sein unvergleichliches Lachen wirkte wie ein Naturwunder. Wir lemten ihn auch als vortrefflichen Koch und Gastgeber kennen - Ochsenschwanz, Knoblauch und kimchi!

Die Türkei sowie türkische Völkerschaften im allgemeinen nahmen in Songmoos Herzen eine Sonderstellung ein. Er besuchte fast jăhrlich die Türkei, speziell das ostliche Anatolien, worüber er interessante Bildreportagen schrieb, so z. B. über die gigantischen Skulpturen von Kommagene.

$\mathrm{Zu}$ den sonstigen wissenschaftlichen Arbeiten Songmoo Khos gehören u.a. ein kleines koreanisches Handbuch über die eurasiatischen Sprachen (1980, 106 S.) und eine Übersicht über die russische Koreanistik (Korean Studies in Russia 1677-1930. Studia Orientalia 51: 12, 1980, 27 S.; auch auf Koreanisch in Hangeul 169, 1980, S. 193-212). In derselben Nummer (S. 151-154) erschien auch eine erstmalige Ausgabe von Ramstedts skizzenartigen Kindheitserinnerungen (»Minä ja maailma»), übersetzt von Songmoo Kho. Er übersetzte auch Ramstedts Reisebericht Sieben Reisen nach Osten ins Koreanische (1983, 248 S.) und versah den Bericht mit einem Index. Seine Ausgabe der von G. J. Ramstedt selbst erstellten, aber nicht veroffentlichten koreanischen Etymologien (Paralipomena of Korean Etymologies, MSFOu 182, 1982, 295 S.) beschreibt er wie folgt: "Paralipomena is a kind of etymological dictionary of Korean with about 1,100 entries. This is not to say that all Ramstedt's ideas 
were correct, nevertheless it should be remembered that the materials published in the present volume still contain many significant insights into the history of the Korean language.» Daneben schrieb er eine beträchtliche Anzahl von gelehrten und populären Artikeln.

G. J. Ramstedt war in mancher Hinsicht der Ausgangspunkt für Songmoos Aktivitäten. Seinen Bemühungen ist es auch zu verdanken, daß dem großen finnischen Gelehrten, »den das koreanische Volk mehr als alle anderen Völker der Welt verehrt», postum der hohe Moran-Orden verliehen wurde.

Im August 1991 nahm Songmoo Kho ein schwieriges Amt in Zentralasien entgegen. Er sollte nun unter den von Stalin zwangsdeportierten Koreanern wirken, über die er ja bereits seine Dissertation geschrieben hatte und welche das wichtigste Quellenmaterial geliefert hatten. Auf persönliche Initiative von Professor Sagymbaj Kabaschevitsch Kosybajev wurde er eingeladen, an der Fakultät für Journalistik (Koreanische Abteilung) der Staatlichen Universität von Kasachstan Koreanisch zu unterrichten. Die praktischen Voraussetzungen für den Koreanisch-Unterricht unter den sowjetkoreanischen Studenten waren so dürftig, daß das Korjo Jezhenedel'nik im Mai 1991 schrieb: "Wir warten auf Wunder und auf Professor Songmoo Kho.»

Seine Aufgabe weitete sich bald aus und bestand nun darin, den Sprachunterricht und die kulturellen Aktivitäten der koreanischen Minorität Kasachstans und Usbekistans zu organisieren und zu koordinieren. Diese Arbeit wurde vom südkoreanischen Staat finanziert. An den Pädagogischen Instituten und einigen anderen Hochschulen arrangierte er Kurse und versuchte daneben, aus eigenem Interesse die kulturellen Verbindungen zwischen der Türkei und Zentralasien zu stärken. Er besuchte Nukus und Ürgentsch und war begeistert, Usbekisch und Kasachisch vor Ort erlernen zu können, - die gefährlichen Umstände bildeten allerdings ein ständiges Risiko.

Um die tragische Siedlungsgeschichte der koreanischen Minorität Zentralasiens weiter erhellen zu können, begründete er eine neue vielsprachige Publikation mit dem Titel News on Korean Studies in Kazakstan and Central Asia (Vorläufer: Бюллетэнь. Общество корееведов Казахстама. Alma Ata 1992: 1). Die Veröffentlichung enthält wichtige Dokumente und seltene Fotografien. Er konnte nur vier Nummern in Helsinki drucken lassen (1993: 1-4); während seines letzten Aufenthaltes in Helsinki im August 1993 erzählte er, daß er jetzt auf der Spur von etwas ganz Sensationellem sei.

Am 21.9.1993 verunglückte unser treuer Freund und Kollege, der außerordentliche Repräsentant des koreanischen Volkes, Songmoo Kho in Kasachstan bei einem verhängnisvollen Autounfall. - Die Besten müssen als erste gehen, sagt man; ein Trost ist das nicht. Songmoo Kho wurde am 1.10.1993 in seinem Heimatort Keum-San beigesetzt.

Songmoo Kho war Mitglied vieler einheimischer und ausländischer wissenschaftlicher Gesellschaften. Besonders aktiv wirkte er in der Association 
for Korean Studies in Europe (AKSE) und der Finnisch-Ugrischen Gesellschaft (Helsinki). Er war auch Präsident der neuen, in Paris residierenden weltweiten Union der Koreanisten. Unter seiner Leitung hielt jene Union i. J. 1990 ihre erste Konferenz in Alma-Ata ab. Er reiste viel, erlebte drei Kontinente, wollte sich aber schließlich in dem Haus des Großvaters als Privatgelehrter niederlassen. Dieser seltene Mensch wird nicht aufhören, in unserem Herzen weiterzuleben.

HARRY HALÉN 